\title{
Modified Single Finite Fourier Cosine Integral Transform Method for Finding the Critical Elastic Buckling Loads of First Order Shear Deformable Beams with Fixed Ends
}

\author{
Charles C. Ike ${ }^{1 *}$, Clifford U. Nwoji², Hyginus N. Onah ${ }^{2}$, Benjamin O. Mama², Michael E. Onyia ${ }^{2}$ \\ ${ }^{1}$ Department of Civil Engineering, Enugu State University of Science and Technology, Enugu 400001, Nigeria \\ ${ }^{2}$ Department of Civil Engineering, University of Nigeria, Nsukka 410001, Nigeria
}

Corresponding Author Email: charles.ike@esut.edu.ng

https://doi.org/10.18280/rcma.290603

Received: 22 September 2019

Accepted: 27 November 2019

\section{Keywords:}

characteristic buckling equation, critical elastic buckling load, eigenvalue problem, first order shear deformation beam theory, modified single finite Fourier cosine integral transform method

\begin{abstract}
This paper proposes a novel modified single finite Fourier cosine integral transform, which determines the elastic buckling loads of moderately-thick, fixed-end beams made of homogeneous, isotropic, linear elastic materials. First, such a beam was modelled with a fourth-order ordinary differential equation, according to the first-order shear deformation theory. Then, the single finite Fourier cosine integral transform was modified to satisfy all the boundary conditions at the fixed ends,using the apriori knowledge, and provide an exact buckling mode (shape) function for the beam. Through the modified transform, the boundary value problem was converted to an algebraic eigenvalue problem, which canbe described by a set of homogeneous algebraic equations. For nontrivial solutions, the characteristic buckling equation was drived from the vanishing of the determinant of the coefficient matrix. Solving the characteristic buckling equation, the authors obtained the eigenvalues and thus derived the buckling loads. The critical buckling load was found to correspond to the first buckling mode. The proposed modified transform gave exact expressions for the buckling loads and the critical buckling load of the fixed ends, because the integral kernel function satisfies all the boundary conditions, and the transform on the domain governing equation satisfies all the domain equations. The solutions of our method agree well with the previous studies.
\end{abstract}

\section{INTRODUCTION}

Several theories have been derived in the literature for beam stability problems, where beams are subjected to axial compressive forces and it is required to determine the loads at which instabilities occur and their modes/shapes [1-5]. The Euler-Bernoulli beam theory (EBBT) derived and developed using the Euler-Bernoulli hypothesis of orthogonality of the beam cross-sections to the middle plane before and after deformation has been extensively used to describe thin beam flexure and stability problems [6, 7]. The implication of the orthogonality assumption of the plane cross-sections to the middle plane is the disregard of transverse shear deformation, hence constraining the scope and use of the theory to thin (slender) beams where elasticity theory prove that transverse shear deformation effects are insignificant [8-13]. The EBBT thus overestimates the buckling load capacities of moderately thick beams since the effects of transverse shear deformation substantially impact the stability behaviour.

Timoshenko derived the Timoshenko beam theory (TBT), a first order shear deformation beam theory by assuming a relaxation of the orthogonality conditions used in formulation of the EBBT [14]. TBT can be formulated using equilibrium methods or variational methods. TBT assumes a constant transverse shear strain across the thickness, thus violating the stress-free boundary conditions on the beam surfaces. It thus requires problem defined shear stress modification factors and is formulated in terms of two unknown displacement functions, rendering the boundary value problem (BVP) complex for analysis and solution.
Further research into buckling analysis have resulted in the derivation and development of higher order shear deformation theories by Krishna Murty, Levinson, Heyliger and Reddy, Ghugal, et al. [13-26].

In this study, the elastic buckling problem of moderately thick beams of prismatic cross-sections, made of homogeneous, isotropic material, modelled as first order shear deformable beam was solved, for the first time, using a modified single (one dimensional) finite Fourier cosine transform method for the case when the beam is fixed at the two ends.

The moderately thick beam studied as shown in Figure 1 for elastic buckling behaviour is defined by $-1 / 2 \leq x \leq 1 / 2$, $-h / 2 \leq y \leq h / 2$ and $-b / 2 \leq z \leq b / 2$. When the cross-section lies in the $y z$ plane and $x$ is the longitudinal axis, $l$ is the beam span, while $h$ is the thickness, $b$ is the width.
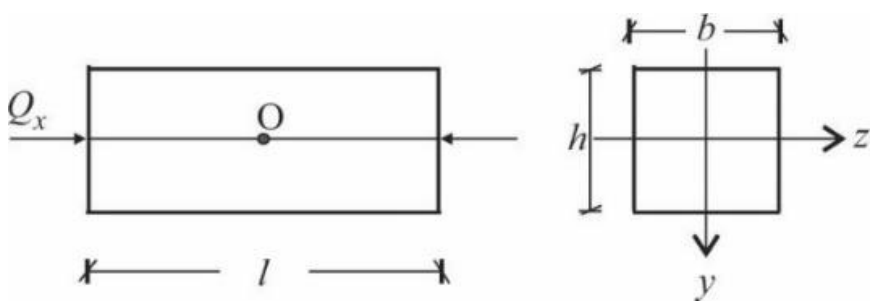

Figure 1. Moderately thick beam studied and the coordinate system 


\section{THEORY}

\subsection{Assumptions}

The assumptions of the formulation are as follows $[8,9]$ :

(i) the transverse displacement field component in the $y$ coordinate direction depends only on the position on the longitudinal coordinate axis of the beam.

(ii) the stress strain equations are one-dimensional.

(iii) the displacements are so small compared to the thickness that the resulting strains are infinitesimal.

(iv) the transverse displacement $v_{t}(x, y)$ is the sum of the bending component $v(x)$ and shear component $v_{s}(x)$.

(v) the material of the beam is homogeneous, linear elastic and isotropic.

\subsection{Displacement components}

The displacement components are:

$$
\begin{gathered}
u_{x}(x, y)=-y \theta(x) \\
u_{y}(x, y)=u_{y}(x)=v(x)+v_{s}(x)
\end{gathered}
$$

where, $\theta(x)$ is the rotation of the cross-section at the neutral axis, $u_{y}(x)$ is the displacement field in the $y$ direction, $v(x)$ is the flexural component of the transverse displacement, and $v_{s}(x)$ is the shear component of the transverse displacement.

$$
\theta(x)=-\frac{d v(x)}{d x}
$$

$y$ is the transverse coordinate direction.

\subsection{Strain field components}

The strain field components are from the small deformation elasticity theory, given by:

$$
\begin{gathered}
\varepsilon_{x x}=\frac{\partial u_{x}}{\partial x}=-y \theta^{\prime}(x) \\
\varepsilon_{y y}=\frac{\partial u_{y}}{\partial y}=0 \\
\gamma_{x y}=\frac{\partial u_{x}}{\partial y}+\frac{\partial u_{y}}{\partial x}=u_{y}^{\prime}(x)-\theta(x)
\end{gathered}
$$

where, $\varepsilon_{x x}, \varepsilon_{y y}$ are normal strains in the $x$ and $y$ directions respectively, $\gamma_{x y}$ is the shear strain and the primes represent derivatives with respect to $x$.

\subsection{Stress fields}

The stress fields for plane stress are from the one dimensional stress-strain relations, given by:

$$
\begin{gathered}
\sigma_{x x}=E \varepsilon_{x x}=-E y \theta^{\prime}(x) \\
\sigma_{y y}=E \frac{\mu}{1-\mu^{2}} \varepsilon_{x x}=-\frac{E \mu}{1-\mu^{2}} y \theta^{\prime}(x)
\end{gathered}
$$

$$
\tau_{x y}=k G \gamma_{x y}=k G\left(u_{y}^{\prime}(x)-\theta(x)\right)
$$

where, $k$ is the shear correction/modification factor, $\sigma_{x x}, \sigma_{y y}$ are the normal stresses, $\tau_{x y}$ is the shear stress, $\mu$ is the Poisson's ratio, $E$ is the Young's modulus of elasticity, $G$ is the shear modulus or the modulus of rigidity.

\subsection{Total potential energy functional, $\Pi$}

The total potential energy functional is given by:

$$
\begin{gathered}
\Pi=\frac{1}{2} \iiint \sigma_{x x} \varepsilon_{x x} d v+\frac{1}{2} \iiint \tau_{x y} \gamma_{x y} d v- \\
\frac{1}{2} \int_{0}^{l} Q_{x}\left(\frac{d u_{y}}{d x}\right)^{2} d x-\int_{0}^{l} q(x) u_{y}(x) d x
\end{gathered}
$$

Using the stress-strain relations, $\Pi$ is given as:

$$
\begin{aligned}
& \Pi=\frac{1}{2} \iiint E \varepsilon_{x x}^{2} d v+\frac{1}{2} \iiint k G \gamma_{x y}^{2} d v- \\
& \frac{1}{2} \int_{0}^{l} Q_{x}\left(\frac{d u_{y}}{d x}\right)^{2} d x-\int_{0}^{l} q(x) u_{y}(x) d x
\end{aligned}
$$

Using the strain-displacement relations, $\Pi$ is simplified as:

$$
\begin{gathered}
\Pi=\frac{1}{2} \iiint E y^{2}\left(\theta^{\prime}(x)\right)^{2} d A d x+ \\
\frac{1}{2} \iiint k G\left(u_{y}^{\prime}(x)-\theta(x)\right)^{2} d A d x- \\
\frac{1}{2} \int_{0}^{l} Q_{x}\left(\frac{d u_{y}}{d x}\right)^{2} d x-\int_{0}^{l} q(x) u_{y}(x) d x
\end{gathered}
$$

Using the Euler-Lagrange condition for the minimization of $\Pi$, the governing stability equation is obtained for rectangular cross-sections with $k=5 / 6$ and $q(x)=0$, as:

$$
\left(E I-Q_{x} \frac{h^{2}}{5}(1+\mu)\right) \frac{d^{4} v(x)}{d x^{4}}+Q_{x} \frac{d^{2} v(x)}{d x^{2}}=0
$$

where, $I$ is the moment of inertia of the beam cross-section, $h$ is the beam thickness.

\section{METHOD}

The one dimensional (single) finite Fourier cosine transform of a function of one independent variable $f(x)$ defined over the finite integral $0 \leq x \leq a$ is denoted by $F_{c}(n)$ and is defined as:

$$
F_{c}(n)=\int_{0}^{a} f(x) \cos \frac{n \pi x}{a} d x
$$

where, $n$ is a nonnegative integer.

The inverse finite Fourier cosine transform of $F_{c}(n)$ is given by: 


$$
f(x)=\frac{1}{a} F_{c}(0)+\frac{2}{a} \sum_{n=1}^{\infty} F_{c}(n) \cos \frac{n \pi x}{a}
$$

By integration by parts, the finite Fourier cosine transform of derivatives of $f(x)$ could be determined.

$$
\begin{gathered}
\int_{0}^{a} \frac{d f(x)}{d x} \cos \frac{n \pi x}{a} d x=\left[f(x) \cos \frac{n \pi x}{a}\right]_{0}^{a}+ \\
\frac{n \pi}{a} \int_{0}^{a} f(x) \sin \frac{n \pi x}{a} d x \\
F_{c}\left(f^{\prime}(x)\right)=(-1)^{n} f(a)-f(0)+\frac{n \pi}{a} F_{s}(n)
\end{gathered}
$$

where, $F_{s}(n)$ is the single finite Fourier sine transform of $f(x)$. If $f(x)$ vanishes at $x=0$, and $x=a$, a simpler expression is obtained for $F_{c}\left(f^{\prime}(x)\right)$ as:

$$
F_{c}\left(f^{\prime}(x)\right)=\int_{0}^{a} f^{\prime}(x) \cos \frac{n \pi x}{a} d x=\frac{n \pi}{a} F_{s}(n)
$$

The expressions for the single finite Fourier cosine transforms of higher derivatives of $f(x)$ are obtained by the repeated application of the fundamental results for the first derivatives.

$$
\begin{aligned}
& F_{c}\left(f^{\prime \prime}(x)\right)=\int_{0}^{a} f^{\prime \prime}(x) \cos \frac{n \pi x}{a} d x= \\
& (-1)^{n} f^{\prime}(a)-f^{\prime}(0)-\left(\frac{n \pi}{a}\right)^{2} F_{c}(n)
\end{aligned}
$$

If $\frac{d f}{d x}=f^{\prime}(x)$ vanishes at the end points $x=0$, and $x=a$, a simpler expression results as follows:

$$
F_{c}\left(f^{\prime \prime}(x)\right)=-\left(\frac{n \pi}{a}\right)^{2} F_{c}(n)
$$

For the fourth order derivative $f^{i v}(x)$, by induction, it is obtained for $f(x)$ vanishing at $x=0$, and $x=a$ that:

$$
\begin{gathered}
F_{c}\left(f^{i v}(x)\right)=\int_{0}^{a} \frac{d^{4}}{d x^{4}} f(x) \cos \frac{n \pi x}{a} d x \\
F_{c}\left(f^{i v}(x)\right)=-\left(\frac{n \pi}{a}\right)^{2} \int_{0}^{a} \frac{d^{2} f(x)}{d x^{2}} \cos \frac{n \pi x}{a} d x=\left(\frac{n \pi}{a}\right)^{4} F_{c}(n)
\end{gathered}
$$

\section{RESULTS}

The governing BVP is represented as the fourth order ordinary differential equation (ODE):

$$
\frac{d^{4} v(x)}{d x^{4}}+\lambda^{2} \frac{d^{2} v(x)}{d x^{2}}=0
$$

where,

$$
\lambda^{2}=\frac{Q_{x}}{E I-\frac{Q_{x} h^{2}(1+\mu)}{5}}
$$

Following the definition of the single finite Fourier cosine integral transform, we modify the Fourier cosine kernel function to ensure that it satisfies the fixed-fixed boundary conditions as:

$$
K(x)=\cos \frac{2 n \pi x}{l}
$$

where, $-l / 2 \leq x \leq l / 2$ and the origin is defined at the middle of the beam.

The single finite Fourier cosine integral transform of the governing BVP becomes:

$$
\int_{0}^{l / 2}\left(\frac{d^{4} v(x)}{d x^{4}}+\lambda^{2} \frac{d^{2} v(x)}{d x^{2}}\right) \cos \frac{2 n \pi x}{l} d x=0
$$

From the linearity property of the finite Fourier cosine integral transform, we have:

$$
\begin{gathered}
\int_{0}^{l / 2} \frac{d^{4} v(x)}{d x^{4}} \cos \frac{2 n \pi x}{l} d x+\int_{0}^{l / 2} \lambda^{2} \frac{d^{2} v(x)}{d x^{2}} \cos \frac{2 n \pi x}{l} d x=0 \\
\left(\frac{2 n \pi}{l}\right)^{4} \int_{0}^{l / 2} v(x) \cos \frac{2 n \pi x}{l} d x- \\
\lambda^{2}\left(\frac{2 n \pi}{l}\right)^{2 / / 2} v(x) \cos \frac{2 n \pi x}{l} d x=0
\end{gathered}
$$

But,

$$
\int_{0}^{l / 2} v(x) \cos \frac{2 n \pi x}{l} d x=v_{c}(n)
$$

where, $v_{c}(n)$ is the single finite Fourier cosine transform of $v(x)$. Then, we have:

$$
\left(\frac{2 n \pi}{l}\right)^{4} v_{c}(n)-\lambda^{2}\left(\frac{2 n \pi}{l}\right)^{2} v_{c}(n)=0
$$

Simplification yields:

$$
\left(\left(\frac{2 n \pi}{l}\right)^{4}-\lambda^{2}\left(\frac{2 n \pi}{l}\right)^{2}\right) v_{c}(n)=0
$$

Simplifying, we have the algebraic eigenvalue problem: 


$$
\left(\left(\frac{2 n \pi}{l}\right)^{2}-\lambda^{2}\right) v_{c}(n)=0
$$

For nontrivial solutions, $v_{c}(n) \neq 0$, and the characteristic buckling equation is found as the system of homogeneous algebraic equations:

$$
\left(\frac{2 n \pi}{l}\right)^{2}-\lambda^{2}=0
$$

The eigenvalues are obtained as:

$$
\lambda^{2}=\left(\frac{2 n \pi}{l}\right)^{2}=4\left(\frac{n \pi}{l}\right)^{2}
$$

The eigenvalues are used to obtain the $n$ buckling loads as follows:

$$
\lambda^{2}=4\left(\frac{n \pi}{l}\right)^{2}=\frac{Q_{x}}{E I-\frac{Q_{x} h^{2}(1+\mu)}{5}}
$$

Solving,

$$
\begin{gathered}
Q_{x}=\frac{4\left(\frac{n \pi}{l}\right)^{2} E I}{1+\frac{4}{5} h^{2}(1+\mu)\left(\frac{n \pi}{l}\right)^{2}} \\
Q_{x}=\frac{4(n \pi)^{2}}{1+0.8 h^{2}(1+\mu)\left(\frac{n \pi}{l}\right)^{2}} \frac{E I}{l^{2}}
\end{gathered}
$$

For $\mu=0.25$,

$$
\begin{gathered}
Q_{x}=\frac{4(n \pi)^{2}}{1+\frac{h^{2}}{l^{2}}(n \pi)^{2}} \frac{E I}{l^{2}} \\
Q_{x_{c r}}=\frac{4 \pi^{2}}{1+\pi^{2} \frac{h^{2}}{l^{2}}} \frac{E I}{l^{2}}=Q_{x}(n=1)
\end{gathered}
$$

For $\mu=0.30$,

$$
\begin{gathered}
Q_{x}=\frac{4(n \pi)^{2}}{1+\frac{26}{25} \frac{h^{2}}{l^{2}}(n \pi)^{2}} \frac{E I}{l^{2}} \\
Q_{x_{c r}}=Q_{x}(n=1)=\frac{4 \pi^{2}}{1+\frac{26}{25} \pi^{2} \frac{h^{2}}{l^{2}}} \frac{E I}{l^{2}}
\end{gathered}
$$

Values of the critical buckling load for the first order shear deformable beam for values of Poisson's ratio $\mu$ given as: $\mu=$
0.25 and for $\mu=0.30$ are calculated using Equations (39) and (41) for various values of $h / l$ and presented in Table 1 and 2, respectively.

Table 1. Critical elastic buckling load $Q_{x c r}$ for first order shear deformable beams with Poisson's ratio $\mu=0.25$ for various values of $h / l$ (fixed ends)

\begin{tabular}{ccc}
\hline$h / l$ & $\begin{array}{c}Q_{x c r}(\mu=0.25) \\
\left(\times E I / l^{2}\right)\end{array}$ & $\begin{array}{c}\text { Exact solution, Onah } \text { et } \\
\text { al. }[17],(\text { for } \mu=0.25) \\
\left(\times E I / l^{2}\right)\end{array}$ \\
\hline 0.01 & 39.43949239 & 39.43949 \\
0.02 & 39.32317593 & 39.32317 \\
0.05 & 38.52778267 & 38.52778 \\
0.1 & 35.93206494 & 35.93206 \\
0.15 & 32.30465 & 32.30465 \\
0.2 & 28.30431997 & 28.30432 \\
0.25 & 24.41686668 & 24.41687 \\
0.3 & 20.90725096 & 20.90725 \\
0.35 & 17.87140938 & 17.87141 \\
0.4 & 15.30683408 & 15.30684 \\
0.45 & 13.16563892 & 13.16564 \\
0.5 & 11.38559297 & 11.3856 \\
\hline
\end{tabular}

Table 2. Critical elastic buckling load $Q_{x c r}$ for first order shear deformable beams with fixed ends with Poisson's ratio $\mu=0.30$ for various values of $h / l$

\begin{tabular}{ccc}
\hline$h / l$ & $\begin{array}{c}Q_{x c r}(\mu=0.30) \\
\left(\times E I / l^{2}\right)\end{array}$ & $\begin{array}{c}\text { Exact solution for } \mu= \\
0.30 \text { Onah } \text { et al. }[17] \\
\left(\times E I / l^{2}\right)\end{array}$ \\
\hline 0.01 & 39.43793697 & 39.43793 \\
0.02 & 39.31699165 & 39.31699 \\
0.05 & 38.49070863 & 38.49071 \\
0.10 & 35.80341588 & 35.80341 \\
0.15 & 32.07153655 & 32.07154 \\
0.20 & 27.98745363 & 27.98745 \\
0.25 & 24.0498529 & 24.04985 \\
0.30 & 20.52111487 & 20.52112 \\
0.35 & 17.48854188 & 17.48854 \\
0.40 & 14.94091706 & 14.94092 \\
0.45 & 12.82375231 & 12.82376 \\
0.50 & 11.07048294 & 11.07049 \\
\hline
\end{tabular}

\section{DISCUSSION}

The elastic buckling problem of first order shear deformable thick and moderately thick beams represented by the fourth order ordinary differential equation, Equation (13) is solved in closed form using the modified single finite Fourier cosine integral transform method for the case of fixed ends. For the elastic buckling problem considered, the integral kernel function for the modified single finite Fourier cosine transform identically satisfies all the geometric and force boundary conditions at the clamped ends.

Another merit of the method is that the modified single finite Fourier cosine transform method can be readily applied to obtain the transform of ordinary derivatives of the function of an independent variable. The modified single finite Fourier cosine transformation is applied to the governing fourth order ODE to obtain Equation (26). The use of the linearity property of the single finite Fourier cosine transform and simplification reduced the problem to an algebraic eigenvalue problem represented by Equation (32). For nontrivial solutions of the 
algebraic eigenvalue problem, the determinant of the coefficient matrix is required to vanish resulting in a characteristic buckling equation given by the system of algebraic equations - Equation (33). The eigenvalues (roots) of the characteristic buckling equation are found for all the $n$ buckling modes as Equation (34). The $n$ eigenvalues are used to find the $n$ buckling loads as Equation (37). For $\mu=0.25$, the $n$ buckling loads are obtained using Equation (38) while for $\mu$ $=0.30$, the $n$ buckling loads are obtained using Equation (40). The critical buckling load $Q_{x c r}$ which is the minimum value of the buckling loads is found to occur at the first buckling mode when $n=1$, and found as Equation (39) when $\mu=0.25$, and Equation (41) for $\mu=0.30$.

Values of the critical buckling load $Q_{x c r}$ for various values of the ratio $h / l$ and for $\mu=0.25$, and $\mu=0.30$ for first order shear deformable beams were calculated and shown in Tables 1 and 2, together with exact results obtained by Onah et al [9] using closed form/analytical solutions of the boundary value problem.

Tables 1 and 2 reveal that the critical elastic buckling load $Q_{x c r}$ obtained by the modified single finite Fourier cosine transform method agreed remarkably well with the exact solutions obtained by Onah et al [9] using analytical/closed form methods of solving the boundary value problem. Tables 1 and 2 further show that for $h / l<0.02$ (for thin (slender) beams) the critical elastic buckling load is approximately equal to the critical buckling load obtained from the Euler Bernoulli beam buckling theory for fixed ends. Tables 1 and 2 also reveal that for $h / l>0.02$ the critical elastic buckling load coefficient decreases with increase in $h / l$, showing that the effect of transverse shear deformation is to significantly reduce the critical elastic buckling load capacity of the beam.

\section{CONCLUSION}

The conclusions of the study are as follows:

(i) The modified single finite Fourier cosine integral transform method is an effective mathematical tool for solving the elastic buckling problem of moderately thick shear deformable beams with fixed ends for homogeneous isotropic materials.

(ii) The integral kernel (nucleus) function of the modified single finite Fourier cosine integral transform satisfies all the geometric and force boundary conditions at the fixed ends and is an exact buckling modal function for the shear deformable beam with fixed ends for the choice of origin of the coordinate system used.

(iii) The modified single finite Fourier cosine integral transform method transforms the governing fourth order ordinary differential equation for the problem to an algebraic eigenvalue problem given by a system of homogeneous algebraic equations.

(iv) For nontrivial solutions of the system of homogeneous algebraic equations, the determinant of the coefficient matrix vanishes, yielding the characteristic buckling equation.

(v) The $n$ roots (zeros) of the characteristic buckling equation are found as the $n$ eigenvalues of the eigenvalue problem.

(vi) The $n$ buckling loads are found from the $n$ eigenvalues, and the minimum elastic buckling load called the critical buckling load was found to correspond to the first buckling mode for which $n=1$. (vii) The modified single finite Fourier cosine integral transform method gave exact expressions for the $n$ buckling loads since the exact buckling mode which identically satisfied all the boundary conditions was used, and the domain governing equation was also satisfied identically.

(viii) For $h / l<0.02$, i.e. thin (slender) beams, the critical elastic buckling load coefficient is approximately the same as the critical elastic buckling load coefficient of EulerBernoulli beam theory.

(ix) For $h / l>0.02$, the critical elastic buckling load coefficient decreases as $h / l$ increases, and the effect of shear deformation is found to substantially reduce the critical buckling loads.

(x) Shear deformation is an important consideration in the evaluation of the critical buckling load capacities of moderately thick beams for realistic designs.

\section{REFERENCES}

[1] Ghugal, Y.M. (2006). A new refined bending theory for thick beam including transverse shear and transverse normal strain effects. Departmental Report Applied Mechanics Department Government College of Engineering Anrangabad, India, 1-96.

[2] Ghugal, Y.M., Sharma, P.V. (2009). A hyperbolic shear deformation theory for flexure and vibration of thick isotropic beams. International Journal of Computational Methods, 6(4): 585-604. https//doi.org/10.1142/S0219876209002017

[3] Ghugal, Y.M., Shimpi R.P. (2000). A trigonometric shear deformation theory for flexure and free vibration of isotropic thick beams. Proceedings of Structural Engineering Convention (SPC 2000) IIT, Bombay Mumbai, India, 255-263.

[4] Heyliger, P.R., Reddy, J.N. (1988). A higher order beam finite element for bending and vibration problems. Journal of Sound and Vibration, 126(2): 309-326. https://doi.org/10.1016/0022-460X(88)90244-1

[5] Ike, C.C. (2018). Fourier sine transform method for the free vibration of Euler - Bernoulli beam resting on Winkler foundation. International Journal of Darshan Institute on Engineering Research and Emerging Technologies (IJDI-ERET), 7(1): 1-6.

[6] Ike, C.C. (2018). Point collocation method for the analysis of Euler - Bernoulli beam on Winkler foundation. International Journal of Darshan Institute on Engineering Research and Emerging Technologies (IJDI-ERET), 7(2) 1-7.

[7] Ike, C.C. (2019). Timoshenko beam theory for the flexural analysis of moderately thick beams - variational formulation and closed form solutions. Tecnica Italiana Italian Journal of Engineering Science, 63(1): 34-45. https//doi.org/10.18280/ti-ijes 630105

[8] Ike, C.C., Ikwueze E.U. (2018). Fifth degree polynomial shape functions for the finite element analysis of clamped simply supported Euler-Bernoulli beam. American Journal of Engineering Research, 7(4): 97-105.

[9] Ike, C.C., Ikwueze, E.U. (2018). Ritz method for the analysis of statically indeterminate Euler Bernoulli beams. Saudi Journal of Engineering and Technology (SJEAT), 3(3): https//doi.org/10.21276/sjeat.2018.3.3.3 
[10] Ike, C.C., Ikwueze, E.U., Ofondu I.O. (2018). Picard's successive iteration method for the elastic buckling analysis of Euler columns with pinned ends. Saudi Journal of Civil Engineering (SJCE), 2(2): 76-88.

[11] Ikwueze, E.U., Ike, C.C., Ofondu, I.O. (2018). Least squares weighted residual method for elastic buckling analysis of Euler column with fixed-pinned ends. Saudi Journal of Civil Engineering (SJCE), 2(2): 110-119.

[12] Karama, M., Abaq, K.S., Mistou S. (2003). Mechanical behaviour of laminated composite beam by new multilayered laminated composite structures model with transverse shear stress continuity. International Journal of Solids and Structures, 40(6): 1523-1546. https://doi.org/10.1016/S0020-7683(02)00647-9

[13] Krishna Murty, A.V. (1984). Towards a consistent beam theory. AIAA Journal, 22(6): 811-816. https://doi.org/10.2514/3.8685

[14] Levinson, M. (1981). A new rectangular beam theory. Journal of Sound and Vibration, 74(1): 81-87. https://doi.org/10.1016/0022-460X(81)90493-4

[15] Mama, B.O., Onyia, M.E., Onah, H.N., Nwoji, C.U., Ike, C.C. (2019). Quintic Hermite polynomial shape functions for the finite element analysis of elastic buckling loads of Euler columns. Nigerian Institute of Electrical and Electronic Engineers (NIEEE) National Enginieering Conference, Nsukka, Nigeria, pp. 240-251.

[16] Ofondu, I.O., Ikwueze, E.U., Ike, C.C. (2018). BubnovGalerkin method for the elastic buckling analysis of Euler columns. Malaysian Journal of Civil Engineering (MJCE), 30(2): 331-346. https//doi.org/10.11113/mjce.v30n2.483

[17] Onah, H.N., Nwoji, C.U., Onyia, M.E., Mama, B.O., Ike, C.C. (2019). On the boundary value problem of first order shear deformation theory of beam buckling - exact solutions. NIEEE (Nigerian Institute of Electrical and Electronic Engineers) National Engineering Conference, Nsukka, Nigeria, pp. 221-239.

[18] Onyia, M.E., Rowland-Lato, E.O. (2018). Determination of the critical buckling load of shear deformable unified beam. International Journal of Engineering and Technology, 10(3): 647-657. https//doi.org/10.21817/ijet/2018/v10.i3/18003026

[19] Onyia, M.E., Nwoji, C.U., Onah, H.N., Mama, B.O., Ike, C.C. (2019) Least squares weighted residual method for the elastic buckling analysis of shear deformable beams. 2019 Maiden Alex Ekwueme Federal University, NdufuAlike Ikwo (AE-FUNAI) Faculty of Engineering and Technology International Conference, pp. 325-333.

[20] Pakhera, K.S., Mitra, M., Shimpi, R.P. (2016). Development of single variable new first - order shear deformation theories for plates and beams. Master of Technology dissertation. Indian Institute of Technology, Bombay, India.

[21] Pakhera, K.S., Shimpi, R.P., Guruprasad, P.J. (2017). Buckling analysis of thick isotropic shear deformable beams. Proceedings of ICTACEM 2017 International Conference on Theoretical, Applied, Computational and Experimental Mechanics, IIT Kharagpur, India, pp. 1-7.

[22] Sayyad, A.S. (2011). Comparison of various refined beam theories for the bending and free vibration analysis of thick beams. Applied and Computational Mechanics, 5(2): 217-230

[23] Sayyad, A.S., Ghugal, Y.M. (2016). Single variable refined beam theories for the bending, buckling and free vibration of homogeneous beams. Applied and Computational Mechanics, 10: 123-138.

[24] Sayyad, A.S., Ghugal, Y.M. (2011). Effect of transverse shear and transverse normal strain on the bending analysis of cross ply laminated beams. International Journal of Applied Mathematics and Mechanics, 7(12): $85-118$

[25] Soldatos, K.P. (1992). A transverse shear deformation theory for homogeneous monoclinic plates. Acta Mechanics, 94: 195-200.

[26] Thai, H.T. (2012). A nonlocal beam theory for bending, buckling and vibration of nano beams. International Journal of Engineering Science, 52: 56-64. https//doi.org/10.1016/j.ijengsci.2011.11.011 